KEK Preprint 97-252

BELLE Preprint 98-1

SAGA-HEP 127

\title{
The monitoring system for the aerogel Cherenkov counter of the BELLE detector 1
}

\author{
M.H.R Khan ${ }^{a}$, A. Murakami af , T. Sumiyoshi ${ }^{b}$, T. Kuniya ${ }^{a}$, I. Adachi ${ }^{b}$,

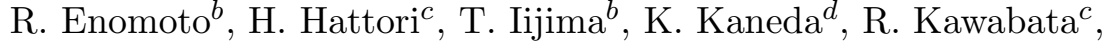 \\ T. Ooba ${ }^{e}$, R. Suda ${ }^{f}$, K. Suzuki ${ }^{c}$ and M. Watanabe ${ }^{e}$ \\ ${ }^{a}$ Department of Physics, Saga University, Saga 840-8502, Japan \\ ${ }^{b}$ Physics Division, High Energy Accelerator Research Organization (KEK), Ibaraki 305-0801, \\ Japan \\ ${ }^{c}$ Department of Physics, Chiba University, Chiba 260-8522, Japan \\ ${ }^{d}$ Applied Fiber Optics Section, Fujikura Limitted, Sakura, Chiba 285-0812, Japan \\ ${ }^{e}$ Department of Physics, Chuo University, Tokyo 112-8551, Japan \\ ${ }^{f}$ Department of Physics, Tokyo Metropolitan University, Tokyo 192-0397, Japan
}

\begin{abstract}
We report on a design and performances of a monitoring system developed for the aerogel Cherenkov counters (ACC) of the BELLE detector. The system consists of blue LEDs, a diffuser box, and optical distributors which distribute the LED light to the ACC modules. The employed LED (NSPB series) has been observed to have high reliability on the long term stability and the temperature dependence. The diffuser box is employed to reduce the intrinsic non-uniformity of the LED light intensity . The overall performances of the present monitoring system on uniformity and intensity of the light output have been found to satisfy all the requirements for the monitoring.
\end{abstract}

Keywords: CP violation, B-factory experiment, particle identification, aerogel Cherenkov counter, gain monitoring.

${ }^{*}$ to be published in Nucl. Instrum. Meth. A

${ }^{\dagger}$ Corresponding author. E-mail address: murakami@cc.saga-u.ac.jp 


\section{Introduction}

Particle identification plays an important role in $\mathrm{CP}$ violation studies in B-factory experiments. A threshold aerogel Cherenkov counter (ACC) system will be used in the BELLE experiment at the KEK B-factory to separate charged pions and kaons in the momentum range from 0.8 to $3.5 \mathrm{GeV} / \mathrm{c}[1,2,3]$. A side view of the aerogel Cherenkov counter of the BELLE detector is shown in Figure 1. The details of the production method of silica aerogel for the BELLE experiment are described in ref.[4].

The ACC consists of 1188 modules and is equipped with a total of 1788 fine-mesh (FM) PMTs to readout the produced Cherenkov photons [5]. A typical single module of the ACC is shown in Figure 2. The FM-PMT is one type of a photo-detector which can be operated in the strong magnetic field [6]. The gain of the FM-PMT and light yield of aerogels may change during the period of data taking. In order to achieve high quality particle identification it is necessary to monitor the gain of PMTs and the light yield of aerogels of the ACC with a precision of a few percent level.

Since monitoring of the output signals from the PMTs of the ACC does not distinguish the change in the gain of the PMTs from that in the light yield of aerogels, we call the monitoring of the PMT gain and the aerogel light yield simply the monitoring of the ACC. The monitoring of these ACC modules can be performed by distributing light pulses from one common light source through optical fibers or by equipping every counter with its own reference light pulser. The former has some advantages over the latter. In the former case we can easily control and monitor the light source itself. To build up such a monitoring system we need a powerful light source and a good light distributing network which can distribute light pulses of almost equal intensity to all of the ACC modules.

The spectrum and timing of the monitoring light should be as similar as possible to that of the Cherenkov light generated by the incident particles. Since the real signal has a few ns rise time, the monitoring light pulse should be as fast as possible. In addition the light pulses should be stable with respect to time and temperature enough to satisfy the requirements of the experiments. Some monitoring systems for calorimeters usually use a Xenon flash lamp[7] , a light emitting diode [8] or a laser[9,10] as a light source. The Xenon flash lamp generates pulses of larger intensity but of wider width than a few hundred $n s$ and the laser is not stable with respect to temperature [10] . An LED is a much more convenient light source of fast and stable pulses. As a light source for the present ACC monitoring system we have chosen the blue LED of NSPB series [11] which has fast response, high intensity, and a wavelength range close to that of the Cherenkov light detected with PMTs.

In the present paper we describe the design, characteristics and the performance of the system to be used to monitor a large number of the ACC modules of the BELLE detector.

\section{General description of the ACC monitoring system}

To monitor the ACC the monitoring system should supply roughly the same light intensity as that of the Cherenkov light generated by the pions to each ACC module, i.e. roughly 18 photoelectrons for each FM-PMT of the ACC module, according to the beam test results [1]. The monitoring light intensity should be stable enough ( $\sim 99 \%)$ with respect to time and temperature to measure the possible drift of the PMT gain and the light yield of aerogels in a

\footnotetext{
${ }^{\ddagger}$ The LED NSPB series is manufactured by NICHIA CHEMICAL INDUSTRIES, Ltd., Anan-shi, Tokushima 774 , Japan.
} 
long period and that due to temperature change. The light distributing network should have small light loss and high uniformity $(\sim 95 \%)$ to supply sufficiently intense and uniform light to all of the 1188 ACC modules. The definition of the stability and the uniformity will be discussed later in Sec.3. In the following subsections we describe each essential component of the present monitoring system.

\subsection{The light source}

From the study of the performance of a Xe-flasher lamp and several blue LEDs, the blue LED NSPB series has been chosen for our monitoring system. All types of LEDs of this series have the same optical characteristics. The LEDs of this series are made of GaN having a single quantum well structure [12]. Due to the optical properties of GaN the LED of NSPB series can emit much higher intensity than other conventional type LEDs available in the market. The peak wavelength of the emission spectrum of the LED of this series is $470 \mathrm{~nm}$ with a FWHM of $20 \mathrm{~nm}$. Its luminous output and luminous intensity are $3.0 \mathrm{~mW}$ and $1.0 \mathrm{~cd}$, respectively at the DC forward voltage of 3.6 volt. The maximum pulse forward current of the LED of this series is $100 \mathrm{~mA}$ and it can be operated with a pulse width up to $10 \mathrm{~ms}$. It has been observed that this LED can be oprerated with pulses of the width at least down to $8 \mathrm{~ns}$. The typical operating temperature is $-20^{\circ} \mathrm{C} \sim+80^{\circ} \mathrm{C}$. The temperature coefficient of the LED of this series will be discussed later. The maximum duty ratio of this type of LED is $1 / 10$.

\subsection{The diffuser box}

The LED light is transmitted to a bundle of 19 pieces of optical fibers (primary fiber bundle). However, when the LED light is directly injected into the fiber bundle, each of 19 fibers will not receive the same light yield because the light intensity distribution of the LED of NSPB series is not uniform in $\theta$ and $\phi$ angle due to the non-symmetrical structure of the light emission part of the LED [13] . To reduce the intrinsic non-uniformity of the light intensity on the input surface of the primary fiber bundle, the LED light is diffused through multiple reflection in a diffuser box. To get uniformly diffused LED light several types of light diffuser boxes having different geometrical structure were studied. The best one which has the highest light intensity was selected. Figure 3 shows the structural view of the present diffuser box. It is made of thin aluminum foil whose inner surface is coated with the $\mathrm{BaSO}_{4}$ reflector. The reflectivity of $\mathrm{BaSO}_{4}$ for the wavelength of $470 \mathrm{~nm}$ was measured to be roughly $98 \%$. The size of the diffuser box is $15 \mathrm{~mm}$ in height having a light injection surface of $18 \mathrm{~mm}$ in diameter. Six LEDs of NSPB500S are attached on the light injection surface of the box as shown in the figure. Emitted light from six LEDs is reflected and diffused by the reflector $\mathrm{BaSO}_{4}$ on the inner surface. The diffused LED light is transmitted to the primary fiber bundle through an out-let hole of an area of 7.1 $m m^{2}$ on the light injection surface. The primary fiber bundle is directly connected to the out-let hole via an optical fiber connector.

\subsection{The Light distributor}

The LED light should be distributed to all ACC modules uniformly with minimum light loss by an optical distributor network. Figure 1 shows one set of the distributor 3 for the present monitoring system. It consists of a primary fiber bundle having 19 primary quartz fibers. Each of these primary fibers has a length of 10 meters and a core/clad diameter of 400/500 $\mu \mathrm{m}$. Fifteen

\footnotetext{
$\S$ The light distributor network is manufactured by Fujikura Limitted, Sakura, Chiba 285, Japan
} 
primary fibers among 19 ones are connected to 15 secondary fiber bundles as indicated in the figure and the other 4 fibers are reserved for spare. Each of the secondary fiber bundles consists of 19 secondary quartz fibers having a length of $0.5 \sim 2.0$ meter and a core/clad diameter of $110 / 125 \mu \mathrm{m}$. For each secondary fiber bundle the 16 secondary fibers are connected to 16 ACC modules one by one and other 3 fibers are for spare .

The diameters of the primary fiber bundle and the secondary fiber bundle are about 3.0 $\mathrm{mm}$ and about $600 \mu \mathrm{m}$, respectively. One set of the distributing network can supply LED light pulses to 240 ACC modules. Four sets of this network monitor all the 960 ACC modules of the barrel part and another one set, 228 modules of the endcap part of the ACC. For the barrel part 4 primary fiber bundles and 64 secondary fiber bundles are prepared; among which 4 secondary fiber bundles are the spare ones. For the endcap part one primary fiber bundle and 17 secondary fiber bundles of the same type as for the barrel part are in fabrication among which 2 secondary fiber bundles are the spare ones. Table 1 shows the statistics of the distributor networks necessary to monitor all of the ACC modules of the BELLE detector.

\subsubsection{The mode scrambler}

Figure 5 shows the structural view of the connection part of the light diffuser box with the primary fiber bundle. The LED light accepted by the primary fiber bundle is transported to the mode scrambler as shown in the figure. The mode scrambler is the several round circular turns of each primary fiber having a radius of $1.75 \mathrm{~cm}$ which is the minimum bending radius of the $10 \mathrm{~m}$ long primary fiber allowed to ensure no light loss in the light transmission. During many revolutions of the light in the mode scrambler the large off-axis angle component of the diffused LED light is filtered out. As a result there is almost no light loss within the $10 \mathrm{~m}$ long primary fiber when the bending curvature of the fiber at any point is larger than $1.75 \mathrm{~cm}$.

\subsubsection{Connection part of the primary fiber with the secondary fiber bundle}

This connection part of the monitoring system affects the uniformity and the intensity of the light transmitted. Thus this connection part is carefully designed and tested. The structural view of this connection part is shown in Figure 6.

The diameter of the secondary fiber bundle is greater than that of each primary fiber. To get the uniform LED light intensity on the input surface of the secondary fiber bundle sufficient space is necessary between the primary fiber and the secondary fiber bundle. The effect of the length of the space on the light intensity and uniformity was studied for two spacers of $2.3 \mathrm{~mm}$ and $3.6 \mathrm{~mm}$ in length between the primary fiber and the secondary fiber bundle. It has been found that the uniformity of light intensity among the secondary fibers are about $95 \%$ and 89 $\%$ for the 3.6 and $2.3 \mathrm{~mm}$ spacing, respectively. The light intensity for the $3.6 \mathrm{~mm}$ spacing is $50 \%$ of that for the $2.3 \mathrm{~mm}$ one. Considering our requirements of light intensity and uniformity the $3.6 \mathrm{~mm}$ spacer is employed between the primary fiber and the secondary fiber bundle as shown in Fig.6.

\section{Performance of the monitoring system}

\subsection{Performance of the LED light source}

The long term stability and temperature dependence of the LED of NSPB series have been studied and are discussed in the following subsections. 


\subsubsection{Experimental arrangement and data taking}

Three pieces of LEDs of NSPB series were selected at random among 100 pieces and were placed in a constant temperature box being kept at constant temperature of $20{ }^{\circ} \mathrm{C}$. Each LED was coupled with an optical quartz fiber of $50 \mu \mathrm{m} \phi$ and $430 \mathrm{~cm}$ length via an optical connector.

During the whole period of measurement the three LEDs were continuously driven with pulses of 5.0 volt in height and of $30 \mathrm{~ns}$ in width at $1 \mathrm{~Hz}$. The continuous driving of the LED at the rate of $1 \mathrm{~Hz}$ for 40 days has supplied the LEDs with $\sim 3.5 \times 10^{6}$ pulses which correspond to driving the same LEDs for about 7 years (assuming that the data taking period for each year is $70 \%$ of a year) with 2000 pulses a day which is expected to be necessary for daily monitoring in the experiment.

Figure 7 shows the setup for the measurement of the stability of the LEDs with respect to time and temperature. The emitted light from the three LEDs, LED1, LED2 and LED3 was led to the light guides of the three scintillation counters, S1, S2 and S3, respectively through the optical fibers. In order to monitor and calibrate the possible drift of the gain of the PMTs used (Hamamatsu H1161), energy losses of cosmic rays in these three scintillation counters $(10 \mathrm{~cm} \times 10 \mathrm{~cm} \times 0.4 \mathrm{~cm})$ were also measured. The cosmic rays were hardened by placing the lead blocks of $5 \mathrm{~cm}$ thickness between the three scintillation counters and the trigger counter $\mathrm{T}$ $(30 \mathrm{~cm} \times 18 \mathrm{~cm} \times 1.0 \mathrm{~cm})$. The whole setup was kept at a constant temperature of $25^{\circ} \mathrm{C}$.

The hardened cosmic rays were triggered by the 4 -fold coincidence of the signals from S1, S2, $\mathrm{S} 3$ and $\mathrm{T}$ counters with a rate of about $1 \mathrm{~Hz}$. The LED and cosmic ray signals were measured simultaneously for about 1 hour every day except 5 days during the whole term (40 days) of the measurements. The output signals from the PMTs were fed to a CAMAC ADC (LeCroy $2249 \mathrm{~W}$ ) and digitized data were recorded with a PC9801 computer. Pedestals were measured before and after every LED/cosmic rays run.

The means of the ADC spectrum for the LED and cosmic ray signals have been obtained by fitting the Gaussian curves to the observed spectra. The number of photoelectrons $N_{p e}$ for each LED and cosmic rays was determined by using the relationship

$$
N_{p e}=\frac{\mu^{2}}{\sigma^{2}}
$$

Here, $\mu$ and $\sigma$ are defined as $\mu=\mu_{\text {obs }}-\mu_{\text {ped }}$ and $\sigma^{2}=\sigma_{\text {obs }}^{2}-\sigma_{\text {ped }}^{2}$, where $\mu_{\text {ped(obs) }}$ and $\sigma_{\text {ped(obs })}$ are the mean and the standard deviation of the ADC spectrum for the pedestal (observed LED or cosmic ray signal), respectively.

\subsubsection{Long term stability of the LED light source}

The ratios of the number of photoelectrons for LEDs to that for cosmic rays, $N_{p e(L E D)} / N_{p e(C o s)}$ at each day are normalized to the averaged values over 35 days and are plotted in Figure 8 as a function of time. It should be noted that the effect of the gain drift of the PMTs is completely cancelled out in this analysis. In the figure the quadratic sum of the statistical errors and the Gaussian-fitting ones is shown.

Assuming the linear variation of the light output from the LEDs the rate of change of light output has been obtained to be $(0.096 \pm 0.045) \%$ /day, $(-0.099 \pm 0.059) \%$ /day and $(-0.28 \pm 0.073) \%$ /day for LED1, LED2 and LED3, respectively. The averaged long term stability for these three LEDs is $(-0.094 \pm 0.035) \%$ /day . It should be noted that in the present measurement one day corresponds to 86400 pulsing of the LED. In the present study the degree 
of stability $\mathrm{S}$ is defined as

$$
S=1-\sqrt{\frac{\sum_{i=1}^{n}\left(x_{i}-\bar{x}\right)^{2}}{(n-1)}} \frac{1}{\bar{x}},
$$

where $x_{i}$ stands for the $\mathrm{i}$-th measured value and $\bar{x}=\sum_{i=1}^{n} x_{i} / \mathrm{n}$. Using this definition the long term stability of the LEDs has been calcuated to be $0.978 \pm 0.007,0.997 \pm 0.006$ and $0.965 \pm 0.006$ for LED1, LED2 and LED3, respectively. The average degree of stability for these three LEDs is $0.980 \pm 0.004$.

The measured stability together with Fig. 8 indicates that LEDs of NSPB series are stable enough for the long-term use for the present monitoring system.

\subsubsection{Temperature dependence of the LED light source}

Using the same experimental setup as the one described in subsection 3.1.1 and changing the temperature in the constant temperature box with a $5{ }^{\circ} \mathrm{C}$ step from $5{ }^{\circ} \mathrm{C}$ to $70{ }^{\circ} \mathrm{C}$, the temperature dependence of the same three LEDs of NSPB series was measured. It should be noted here that the ACC will be kept at around $25^{\circ} \mathrm{C}$ in the BELLE experiment. The LEDs were kept at each temperature for 2 hours to make sure that the LEDs had completely reached the setting temperature. The temperature near the surface of the LEDs was measured continuously with a thermometer and it was observed that within 15 minutes the temperature near the surface of the LED reached the setting temperature.

The ratios of the number of photoelectrons for the LEDs to that for cosmic rays, $N_{p e(L E D)} / N_{p e(C o s)}$ at each temperature are normalized to the averaged values over $5{ }^{\circ} \mathrm{C}$ to $70{ }^{\circ} \mathrm{C}$ and are plotted in Figure 9 as a function of temperature. Fitting the function $\exp (\alpha \mathrm{T})$ to these data in the temperature range from $5^{\circ} \mathrm{C}$ to $70^{\circ}$ for each LED the temperature coefficient $\alpha$ is obtained to be $(0.029 \pm 0.048) \% /{ }^{\circ} \mathrm{C},(-0.067 \pm 0.057) \% /{ }^{\circ} \mathrm{C}$ and $(0.029 \pm 0.074) \% /{ }^{\circ} \mathrm{C}$ for LED1, LED2 and LED3, respectively. The average temperature coefficient for these three LEDs is $(-0.003 \pm 0.035) \% /{ }^{\circ} \mathrm{C}$. This temperature dependence is very small compared with the other LED light sources used in other experiments. The measured temperature dependence indicates that LEDs of NSPB series can be reliably used for the ACC monitoring system. In Table 2 comparison of the performances is shown between the present LED light source and the other ones. It has been shown that the present LED light source has a much better performance than the others.

\subsection{Performance of the diffuser box}

The $\phi$-angular distributions of the light intensity for the non-diffused and diffused LED light were measured. The light emission axis of the LED was defined as the z-axis for the non-diffused LED light and the light intensity distribution with respect to this axis was measured with a $45^{\circ}$ step. The same measurement was performed for the diffused LED light. In this case the center axis of the light out-let hole of the diffuser box is defined as the z-axis. Figure 10 shows the observed $\phi$-angular distributions of the light intensity for the non-diffiused and diffused LED light. By using the diffuser box the uniformity of the $\phi$-angular distribution of the light intensity for the non-diffused LED light was improved from $66.0 \%$ to $99.1 \%$. This number indicates that the diffused light has the complete uniform distribution both in $\theta$ and $\phi$.

It has been observed that the light intensity of the diffused LED light reduces by a factor of $\sim 375$ from that of the non-diffused LED light. The absoluate value of the light intensity through the whole monitoring system with the diffuser box will be discussed in subsection 3.4. 


\subsection{Performance of the distributor}

In order to study the performance of the distributor network the uniformity of light intensity among the fibers of each primary and secondary fiber bundles was measured. The uniformity is defined as

$$
\text { Uniformity }=1-\frac{\sigma}{\bar{\mu}},
$$

where $\bar{\mu}$ is the average of $N_{p e}$ over 19 fibers of each primary or secondary fiber bundle and $\sigma$ is the standard deviation of the distribution of $N_{p e}$ for 19 fibers.

To study the uniformity of light intensity from all the secondary fibers an LED was driven by the square pulse of height/width of $4.0 \mathrm{~V} / 30 \mathrm{~ns}$ at $500 \mathrm{~Hz}$. The LED was directly (without using the diffuser box) coupled to a given primary fiber of a core/clad diameter of 400/500 $\mu \mathrm{m}$ with a space gap of $2.0 \mathrm{~cm}$. The non-diffused LED light from the given primary fiber was transmitted to the secondary fiber bundle. It was confirmed that the non-uniformity of light intensity can be completely neglected on the input surface of the given primary fiber. The light output from each secondary fiber was measured with a line focus PMT of Hamamatsu R329-05S. The output signal of the PMT was read with a CAMAC ADC (LeCory 2249W). Using Eq.(1) the number of photoelectrons $N_{p e}$ for each secondary fiber was calculated.

Measurements of the performance have been made only on the distributors required for the barrel part ACC. The measured uniformity for the 64 secondary fiber bundles is shown in Figure 11. The average of the uniformity is $94.96 \pm 0.10 \%$.

In order to measure the uniformity of light intensity among the primary fibers of each primary fiber bundle the diffused LED light was injected to the input surface of each primary fiber bundle. The light output from each primary fiber was transmitted to a given secondary fiber bundle successively. The light output from one of the given secondary fiber was fed directly to the PMT. The number of photoelectrons was calculated as described above. The uniformity among 19 primary fibers of each primary fiber bundle was calculated using Eq.(3).

The averaged uniformity over the four primary fiber bundles has been measured to be $97.13 \pm 0.32 \%$.

\subsection{Light intensity and uniformity of the whole monitoring system}

Measurements were made on the performance of the whole monitoring system consisting of the LED light source, the diffuser box and the distributor. The six LEDs in the diffuser box were driven with a square pulse of height/width of $5.0 \mathrm{~V} / 30 \mathrm{~ns}$ at $500 \mathrm{~Hz}$. It was confirmed that the LED light output does not depend on the repetition rate of the LED driving pulse in the range from $1 \mathrm{~Hz}$ to $10 \mathrm{KHz}$. The diffused LED light was transmitted to the primary fiber bundle through the out-let hole of the diffuser box. The light output from each of the 19 primary fibers was transmitted to a secondary fiber bundle successively. For each primary fiber bundle the light intensity from each secondary fiber of the secondary fiber bundle was measured by using the line focus PMT R329-05S through the ACC module instead of using a FM-PMT. The same measurement was performed for all of the 4 primary fiber bundles. Using Eq.(1) the number of photoelectrons $N_{p e}$ for each secondary fiber was calculated. Figure 12 shows the observed number of photoelectrons averaged over 19 secondary fibers for each primary fiber. This figure indicates that by adjusting the LED driving voltage for each diffuser box roughly the same light intensity can be supplied to each ACC module.

The light intensity through the whole monitoring system was measured for the LED driving voltage from $3.5 \mathrm{~V}$ to $5.75 \mathrm{~V}$ and for the pulse width from $20 \mathrm{~ns}$ to $60 \mathrm{~ns}$. In Figure 13 the measured number of photoelectrons is shown as a function of the LED driving voltage. This 
figure shows that just controlling the LED driving voltage and/or pulse width we can easily change the number of photoelectrons in the range $10 \sim 40$. It should be noted that the quantum efficiency of the FM-PMT of the ACC is about $25 \%$ at $400 \mathrm{~nm}$ [17] which is nearly the same as that of the PMT R329-05S used in the present measurement. Therefore the same number of photoelectrons given above, i.e. roughly $10 \sim 40$ will be obtained when this monitoring system is employed in the ACC with the FM-PMT. This shows that the developed monitoring system satisfies the requirements of the light intensity for the ACC monitoring which are described in Sec.2.

From the uniformity averaged over the 4 primary and the 64 secondary fiber bundles which are given in Sec.3.3 the non-uniformity of light intensity for the whole system can be obtained using the relation

$$
(N U)^{2}=\left(N U_{p}\right)^{2}+\left(N U_{s}\right)^{2}
$$

where $N U_{p}$ and $N U_{s}$ stand for the non-uniformity averaged over the 4 primary fiber bundles and that over the 64 secondary fiber bundles, respectively. NU is the non-uniformity over the whole monitoring system for the barrel part ACC.

The non-uniformity for the whole monitoring system for the barrel part ACC was then calculated to be $5.80 \pm 0.09 \%$. This means that the present monitoring system has the uniformity of $94.20 \pm 0.09 \%$ over all of the ACC modules of the barrel ACC.

\section{Conclusions}

We have developed a new monitoring system to be used to monitor the ACC modules of the BELLE detector at the KEK B-factory. The employed LED light source has been observed to have the long term stability of $98.0 \pm 0.4 \%$. The temperature coefficient of the LED has been measured to be $(-0.003 \pm 0.035) \% /{ }^{\circ} \mathrm{C}$ over a temperature range of $5{ }^{\circ} \mathrm{C}$ to $70{ }^{\circ} \mathrm{C}$. In this monitoring system the diffuser box is employed to reduce the intrinsic directional non-uniformity of the LED light intensity. The uniformity of light intensity of the developed monitoring system has been measured to be $94.20 \pm 0.09 \%$. The present monitoring system can supply the light intensity corresponding to roughly $10 \sim 40$ photoelectrons to each ACC module. The performances of the present monitoring system meets all the requirements for monitoring of all the ACC modules of the BELLE detector.

\section{Acknowledgment}

This work was partly supported by Grant-in-Aid for Scientific Research on Priority Areas (Physics of CP violation) from the Ministry of Education, Science, and Culture of Japan. We gratefully thank all of the members of the ACC subgroup of the BELLE collaboration for joining in discussions, giving comments and advises on this work. 


\section{References}

[1] T. Iijima et al., Nucl. Instrum. Meth. A379(1996)45\%.

[2] M. Hazumi, Nucl. Phys. B(Proc. suppl.) 59 (1997)61.

T Nozaki, Nucl. Phys. B(Proc. suppl.) 50 (1996)288.

[3] BELLE Collaboration, "Technical Design Report", KEK procedings 95-1.

[4] I. Adachi et al., Nucl. Instrum. Meth. A355 (1995)390.

[5] T. Iijima et al., Nucl. Instrum. Meth. A387(1997)64.

[6] R. Enomoto et al., Nucl. Instrum. Meth. A332 (1993)129.

[7] F. Bonutti et al., Nucl. Instrum. Meth. A337 (1993)165.

T. Sumiyoshi et al., Nucl. Instrum. Meth. A271 (1988)432.

[8] D. Autiero et al., Nucl. Instrum. Meth. A372 (1996)556.

Janusz Zabierowski, Nucl. Instrum. Meth. A388 (1994)577.

J. Berger et al., Nucl. Instrum. Meth. A279 (1989).

Ronald J. Madaras, Barrie Pardoe and Ruben Pecyner, Nucl. Instrum. Meth. 160 (1979)263.

G. Anton, K. Buchler and M. Kuckes, Nucl. Instrum. Meth. A274 (1989)222.

[9] OPAL Collaboration, Nucl. Instrum. Meth. A305 (1991)275.

[10] See for example, S. Bianco et al., Nucl. Instrum. Meth. A305 (1991)48.

[11] Catalogue of NICHIA Chemical Industries Ltd., Japan, Cat. No. 9603.

[12] S. Nakamura, M. Senoh, N. Iwasa and S. Nagahama, Jpn. J. Appl. Phys. 34, Part2, (1995)L797.

S. Nakamura, M. Senoh, N. Iwasa, S. Nagahama, T. Yamada and T. Mukai, Jpn. J. Appl. Phys. 34, Part2, (1995)L1332.

[13] Private communication with NICHIA Chemical Industries Ltd., Japan.

[14] T. Peitzmann et al., Nucl. Instrum. Meth. A376 (1996)368.

[15] G. Anton, K. Buchler and M. Kuckes, Nucl. Instrum. Meth. A274 (1989)222.

[16] BELLE Collaboration, "BELLE Progress Report" 1995 April-1996 March, p. 63.

[17] PMT Catalogue of Hamamatsu Photonics K.K., TPMH 1110E01, Oct., 1995. 


\section{Figure Captions}

Fig.1: Side veiw of the aerogel Charenkov counter system of the BELLE detector. The refractive index $n$ of aerogel is shown for each type of modules.

Fig.2: A typical single module of the aerogel Cherenkov counter. The monitoring light is fed to the ACC module from the light distributor through the fiber connector.

Fig.3: The structural view of the diffuser box(not in scale). Emitted light from 6 LEDs is reflected and diffused by the reflector $\mathrm{BaSO}_{4}$ on the inner surface. The diffused LED light is transmitted to the primary fiber bundle through an out-let hole on the light injection surface.

Fig.4: One complete set of the light distributor network for the BELLE ACC monitoring system. Each primary quartz fiber has a length of $10 \mathrm{~m}$. The secondary quartz fibers has a length of $0.5 \sim 2.0 \mathrm{~m}$.

Fig.5:The structural view of the connection part of the diffuser box with the primary fiber bundle (not in scale). The mode scrambler is several circular turns of each primary fiber having a radius of $1.75 \mathrm{~cm}$.

Fig.6:The structural view of the connection part of the primary fiber with the secondary fiber bundle (not in scale). To get the uniform LED light intensity on the input surface of the secondary fiber bundle $3.6 \mathrm{~mm}$ spacing is used between the primary fiber and the secondary fiber bundle.

Fig.7:Experimental setup for the stability measurement of the LEDs of NSPB series (not in scale). In order to monitor and calibrate the possible gain drift of the PMTs, the energy losses of cosmic rays in S1, S2 and S3 was measured. Light from three LEDs was fed to the light guide of the scintilation counters with three optical fibers.

Fig.8:Long term stability of the LEDs of NSPB series. The ratios of the number of photoelectrons for the LED to that for cosmic rays, $N_{p e(L E D)} / N_{p e(C o s)}$ normalized to the averaged values over 35 days are plotted as a function of time. Only statistical and Gaussian fitting errors are shown.

Fig.9:Temperature dependence of the light intensity of the LEDs of NSPB series. The ratios of the number of plotoelectrons for the LED to that for cosmic rays, $N_{p e(L E D)} / N_{p e(C o s)}$ are normalized to the averaged values over $5{ }^{\circ} \mathrm{C}$ to $70^{\circ} \mathrm{C}$. Only statistical and Gaussian fitting errors are shown.

Fig.10: $\phi$-angle distribution of the non-diffused and diffiused LED light. The $\phi$-angle at which the intensity is maximum is defined as zero degree. All the data points are normalized to the number of photoelectrons obtained at zero degree. Only statistical errors are shown in the figure.

Fig.11:Uniformity of light intensity of the 64 secondary fiber bundles. Each data point shows the uniformity among the 19 secondary fibers of each secondary fiber bundle. Only statistical and Gaussian fitting errors are shown.

Fig.12:The light yield through the whole monitoring system for each primary fiber. Each data point shows the number of photoelectrons averaged over 19 secondary fibers of the given secondary fiber bundle. The error bars are within the circle of the data points.

Fig.13: LED driving voltage vs the light intensity of the LED of NSPB series through the whole monitoring system. The 6 LEDs in the diffuser box were driven with square pulses of height from $3.5 \mathrm{~V}$ to $5.5 \mathrm{~V}$ and of width from $30 \mathrm{~ns}$ to $60 \mathrm{~ns}$. Only statistical errors are shown in the figure. The error bars are within the circle of the data points. 
Table 1: Statistics of the light distributor network for the BELLE ACC monitoring system.

\begin{tabular}{lll}
\hline \hline Parameters & $\begin{array}{l}\text { Primary fiber } \\
\text { (barrel+endacp) }\end{array}$ & $\begin{array}{l}\text { Secondary fiber } \\
\text { (barrel+endcap) }\end{array}$ \\
\hline Number of sets & $(4+1)$ & $(4+1)$ \\
Number of used bundles/set & $(1+1)$ & $(15+15)$ \\
Number of spare bundles & $(0+0)$ & $(64+17)$ \\
Total number of bundles & $(4+1)$ & $(16+16)$ \\
Number of used fibers/bundle & $(15+15)$ & $(3+3)$ \\
Number of spare fibers/bundle & $(4+4)$ & $(285+285)$ \\
Total number of fibers/set & $(19+19)$ & $(1024+240)$ \\
Total number of used fibers & $(60+15)$ & $(1216+323)$ \\
Total number of fibers & $(76+19)$ & $600 \mu m$ \\
Diameter of a fiber bundle & $3.0 \mathrm{~mm}$ & $0.5 \sim 2.0 \mathrm{~m}, 110 / 125 \mu \mathrm{m}$ \\
Length/diameter of each fiber & $10 \mathrm{~m}, 400 / 500 \mu \mathrm{m}$ & \\
\hline \hline
\end{tabular}

Table 2: Comparison of the temperature dependence between the present LED of NSPB series and some other types of LEDs.

\begin{tabular}{llll}
\hline \hline Experiment & LED type/Material & $\begin{array}{l}\text { Temperature } \\
\text { range }\left({ }^{\circ} \mathrm{C}\right)\end{array}$ & $\begin{array}{l}\text { Temperature } \\
\text { coefficient }\left(\% /{ }^{\circ} \mathrm{C}\right)\end{array}$ \\
\hline Present study & blue/GaN & $5 \sim 70$ & $-0.003 \pm 0.035$ \\
WA98[14] & blue/SiC & $10 \sim 60$ & -1.1 \\
PHOENICS[15] & blue & & $-1.0 \pm 0.05$ \\
CsI(BELLE)[16] & blue & $23-31$ & -0.7 \\
\hline \hline
\end{tabular}




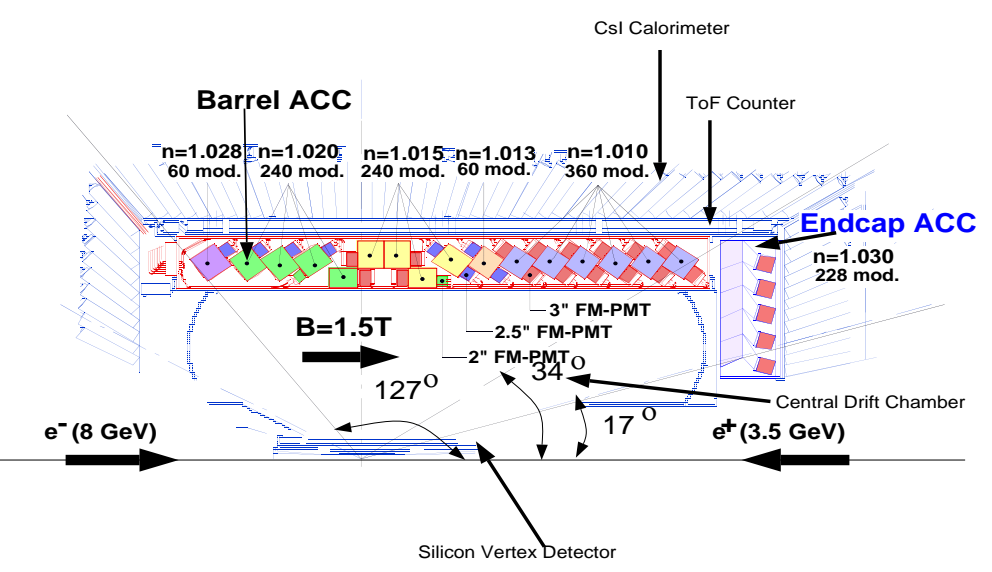

Figure 1: 
Fiber connector for monitoring

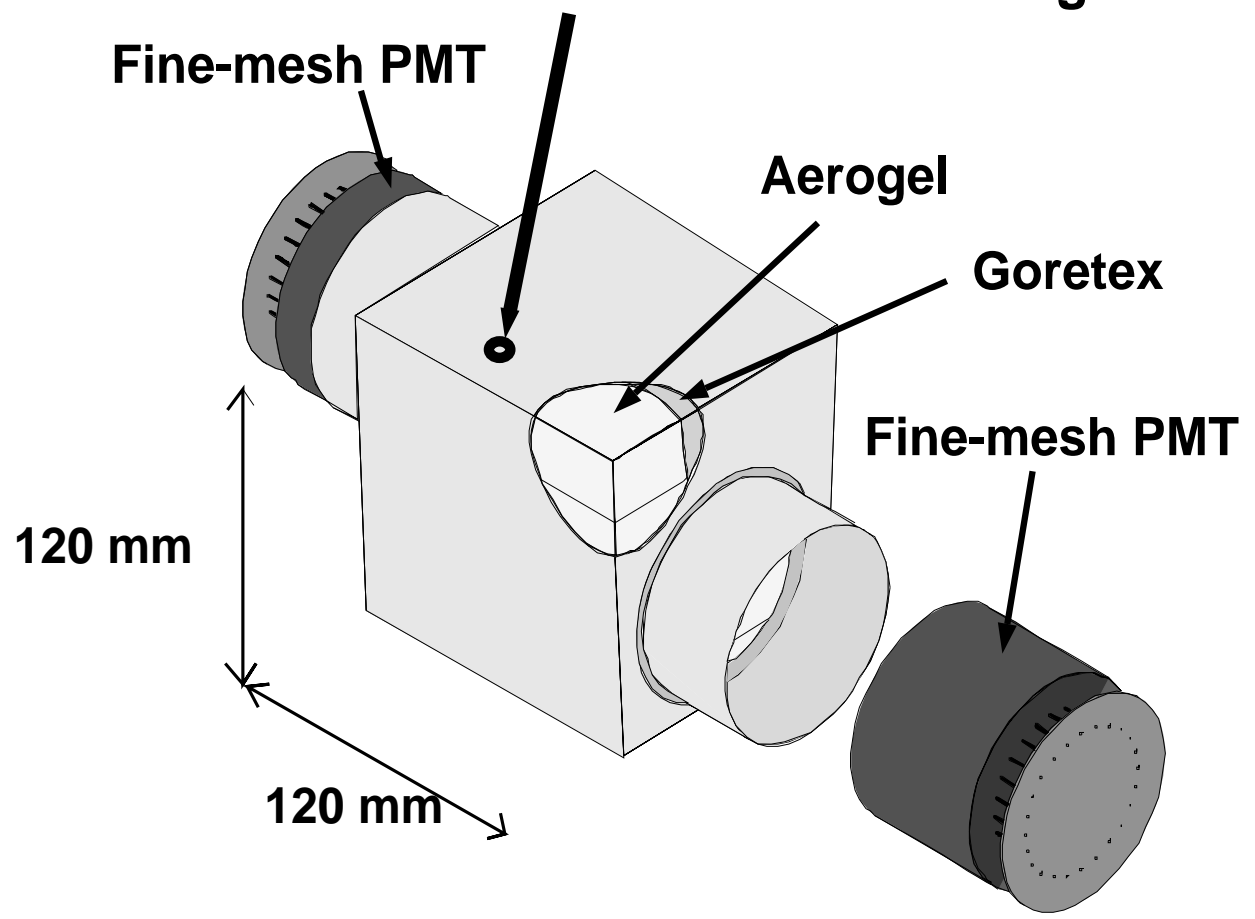

Figure 2: 


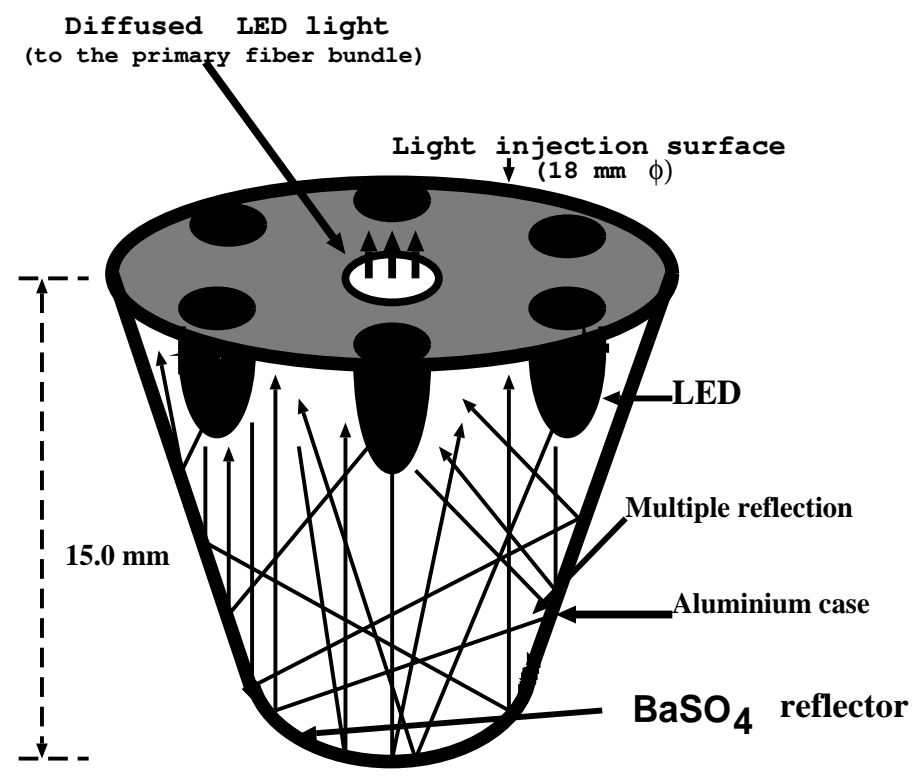

Figure 3: 


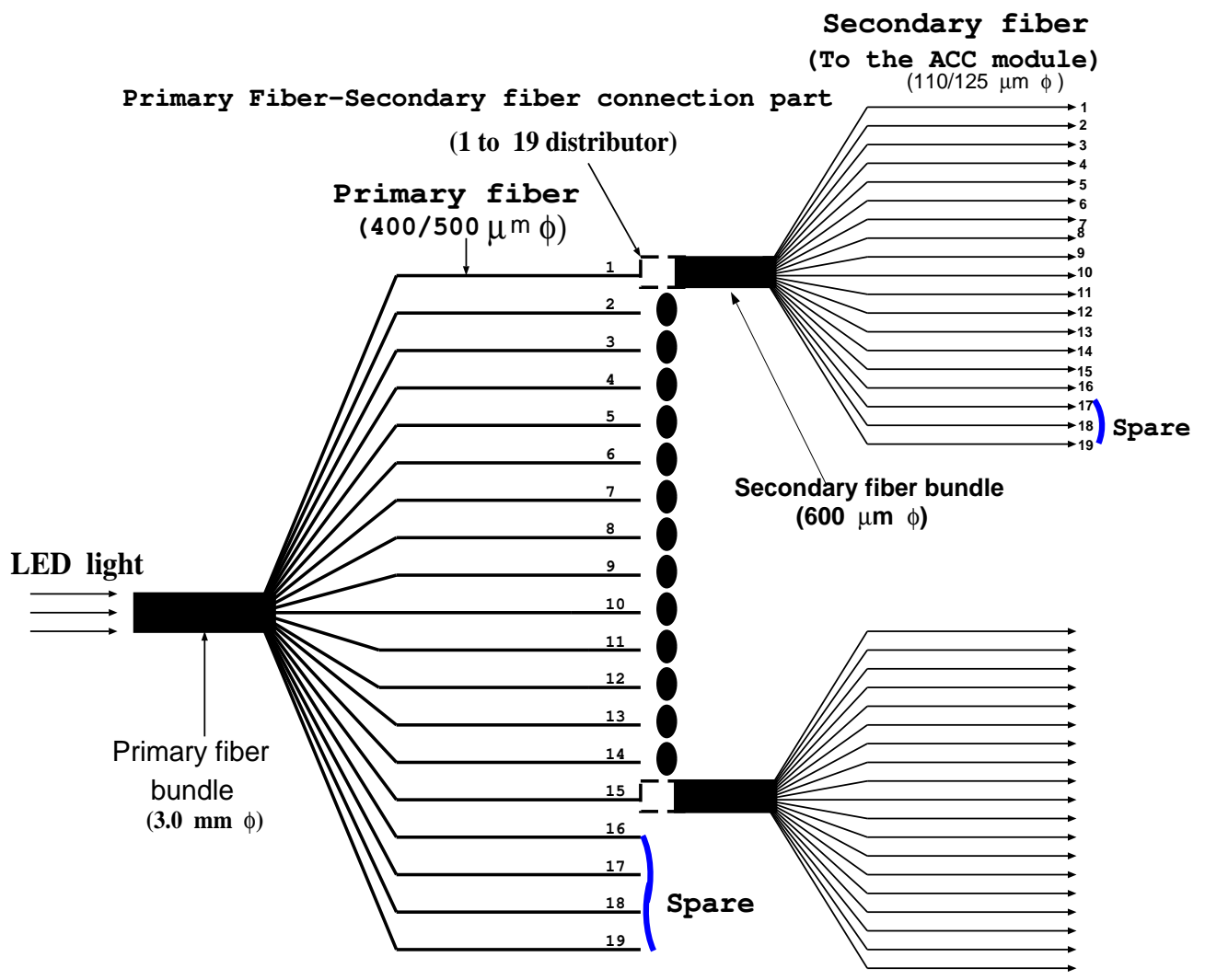

Figure 4: 


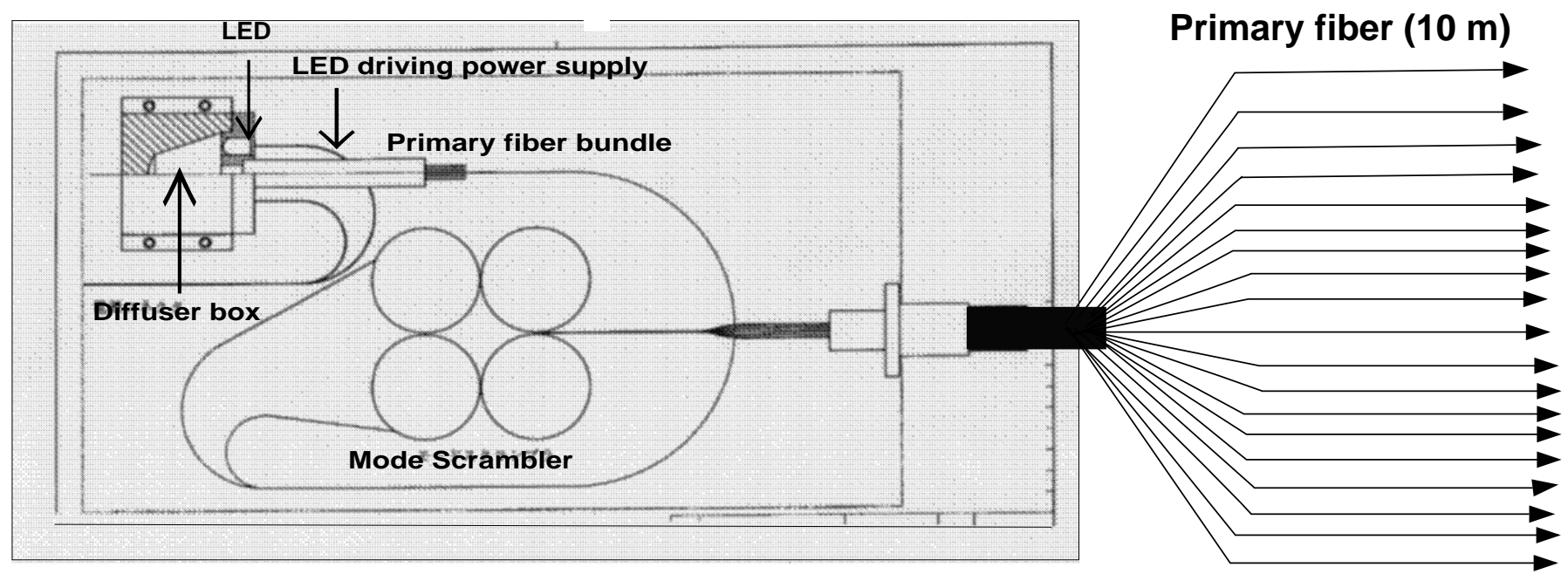

Figure 5: 


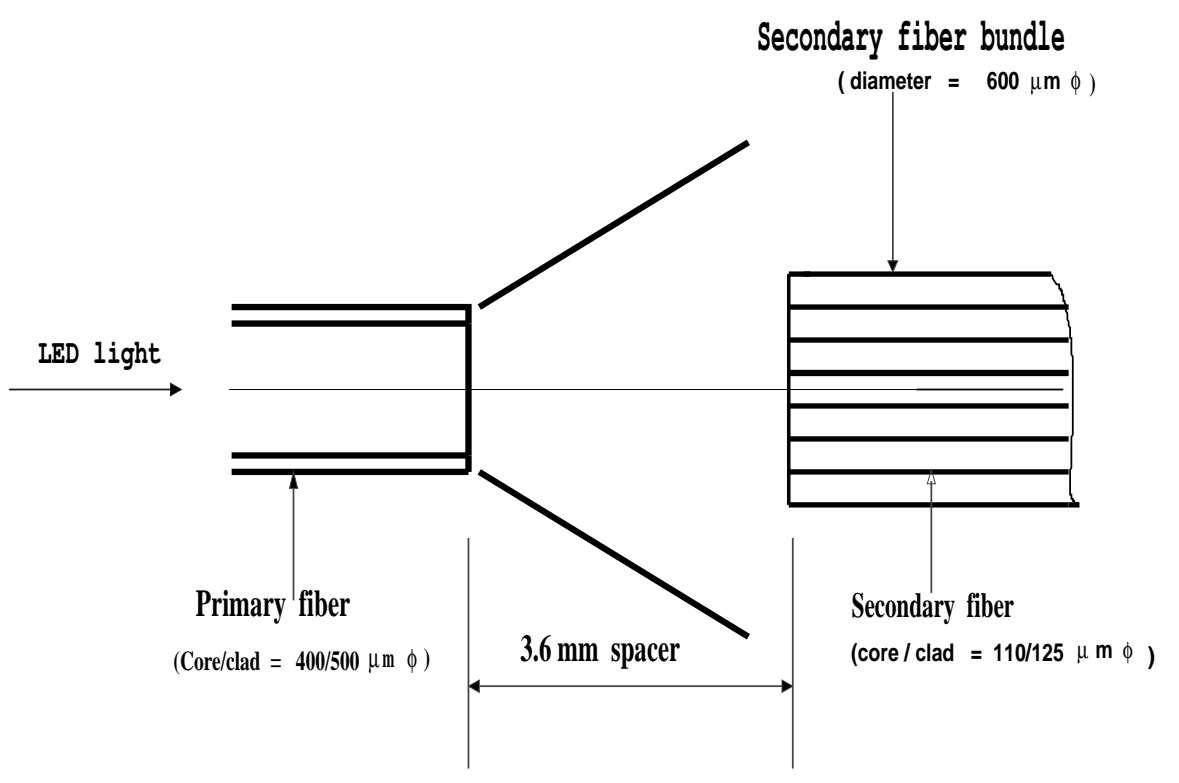

Figure 6: 


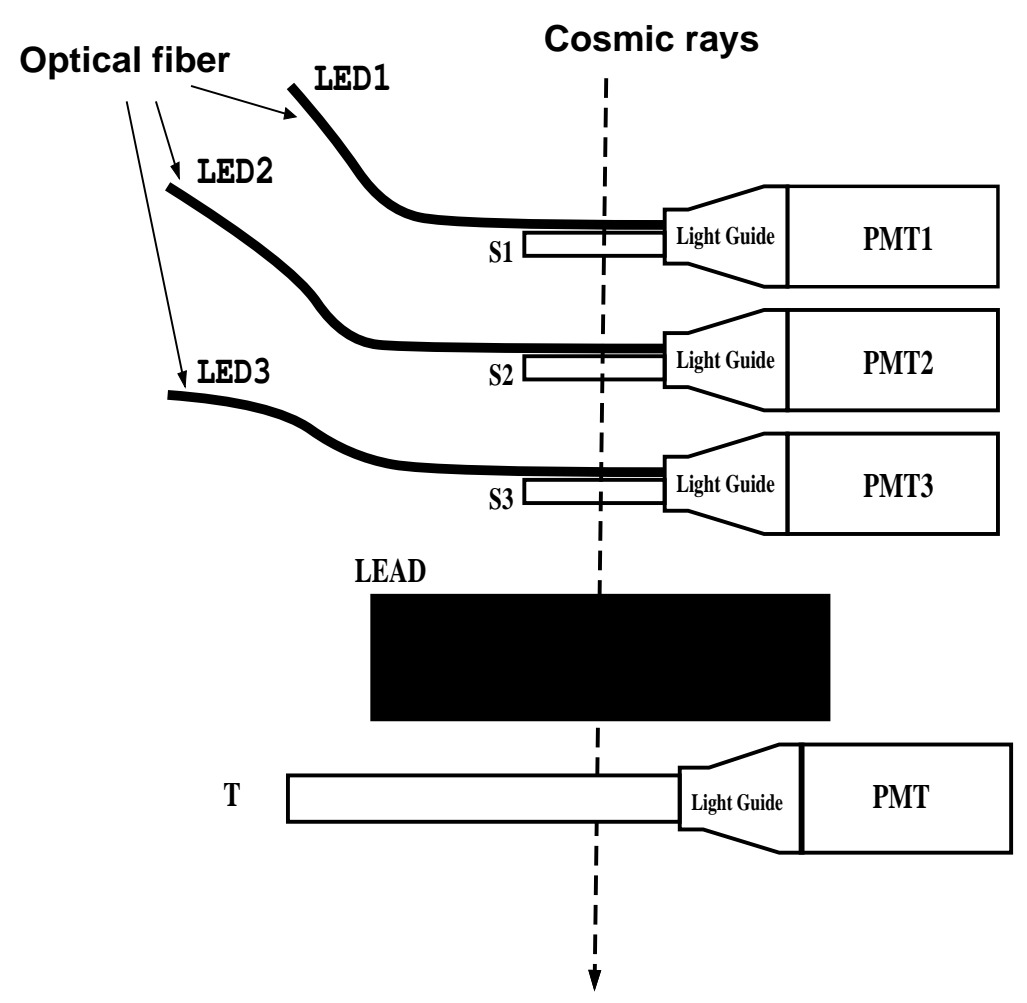

Figure 7: 


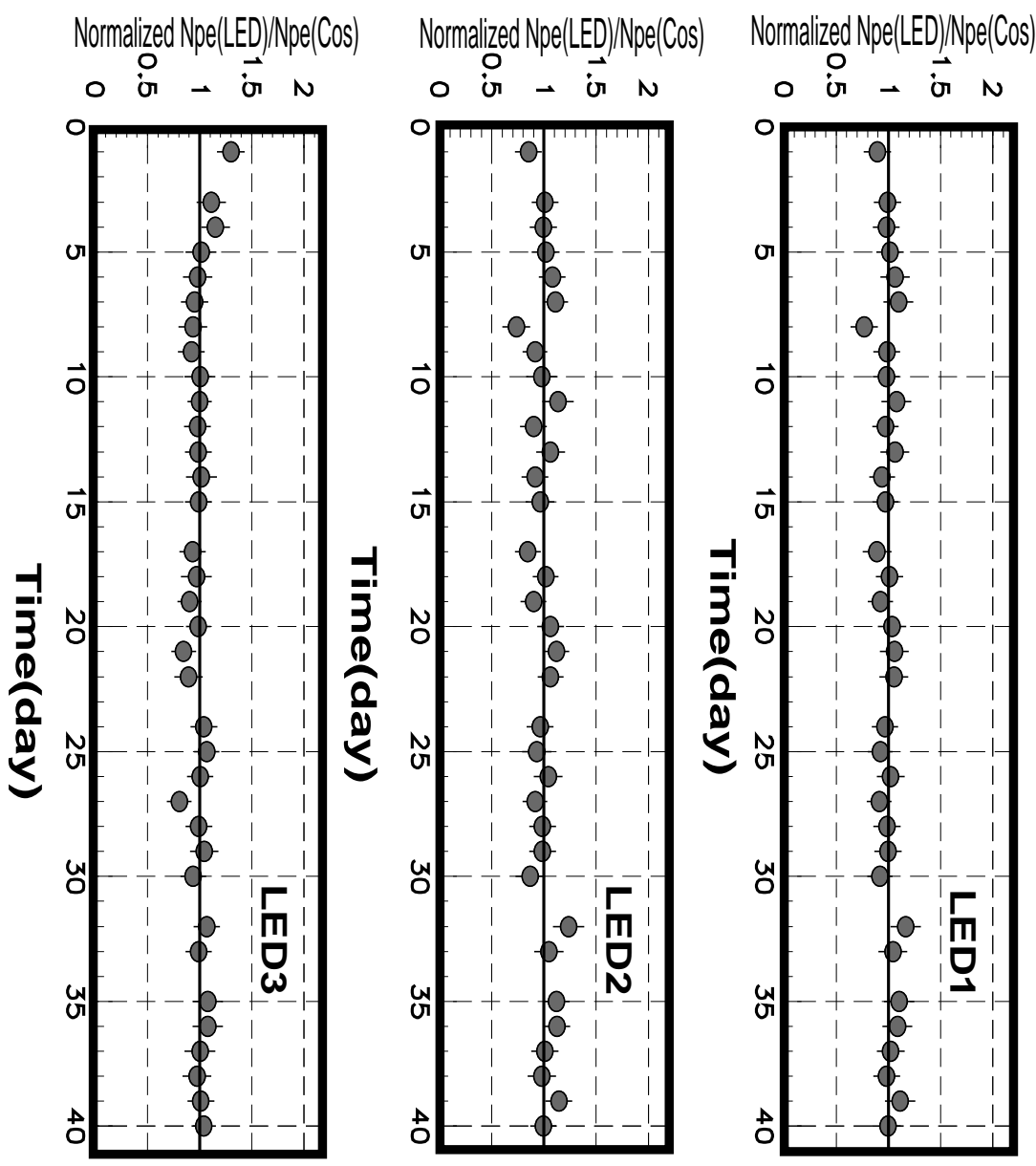



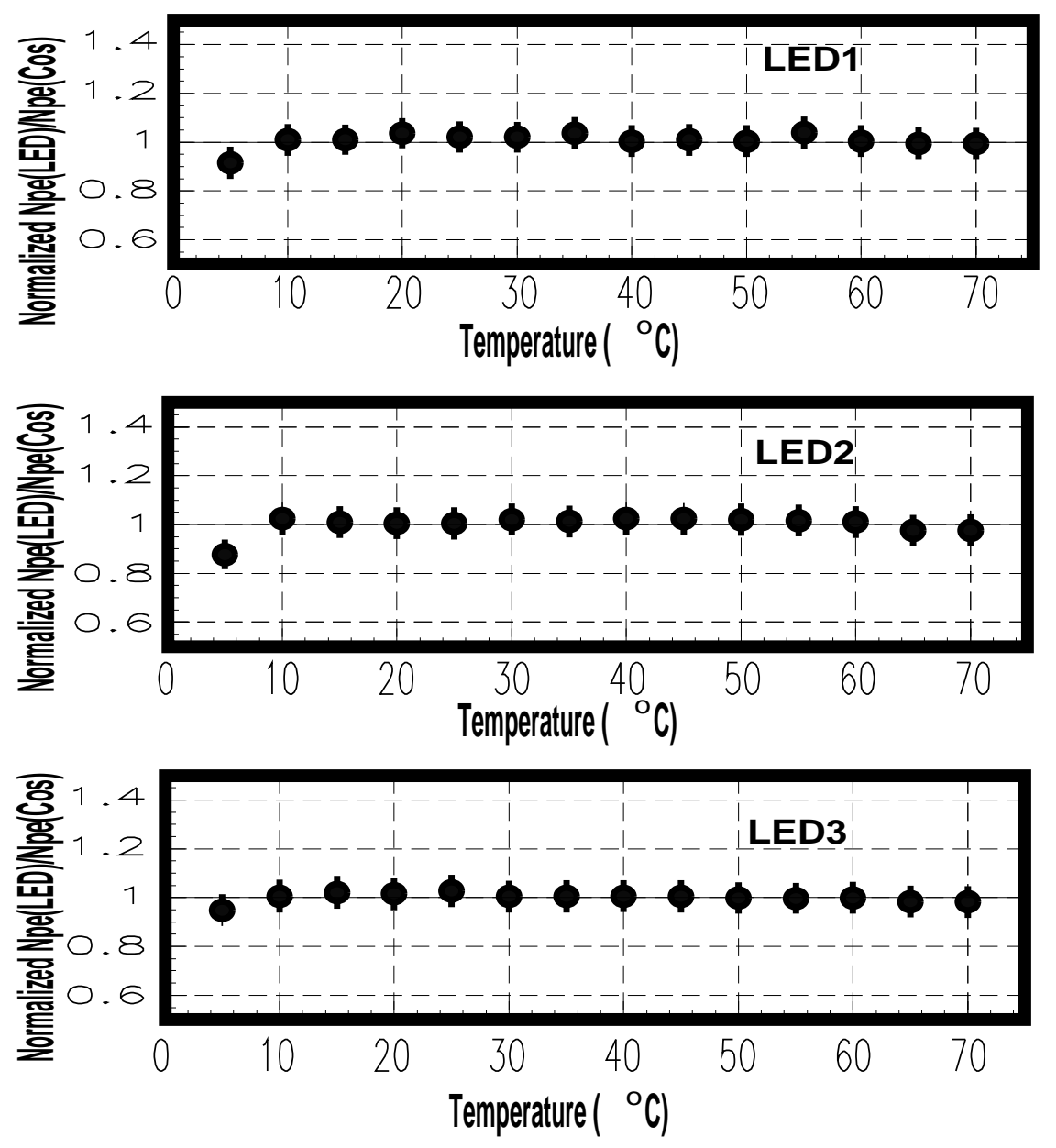

Figure 9: 


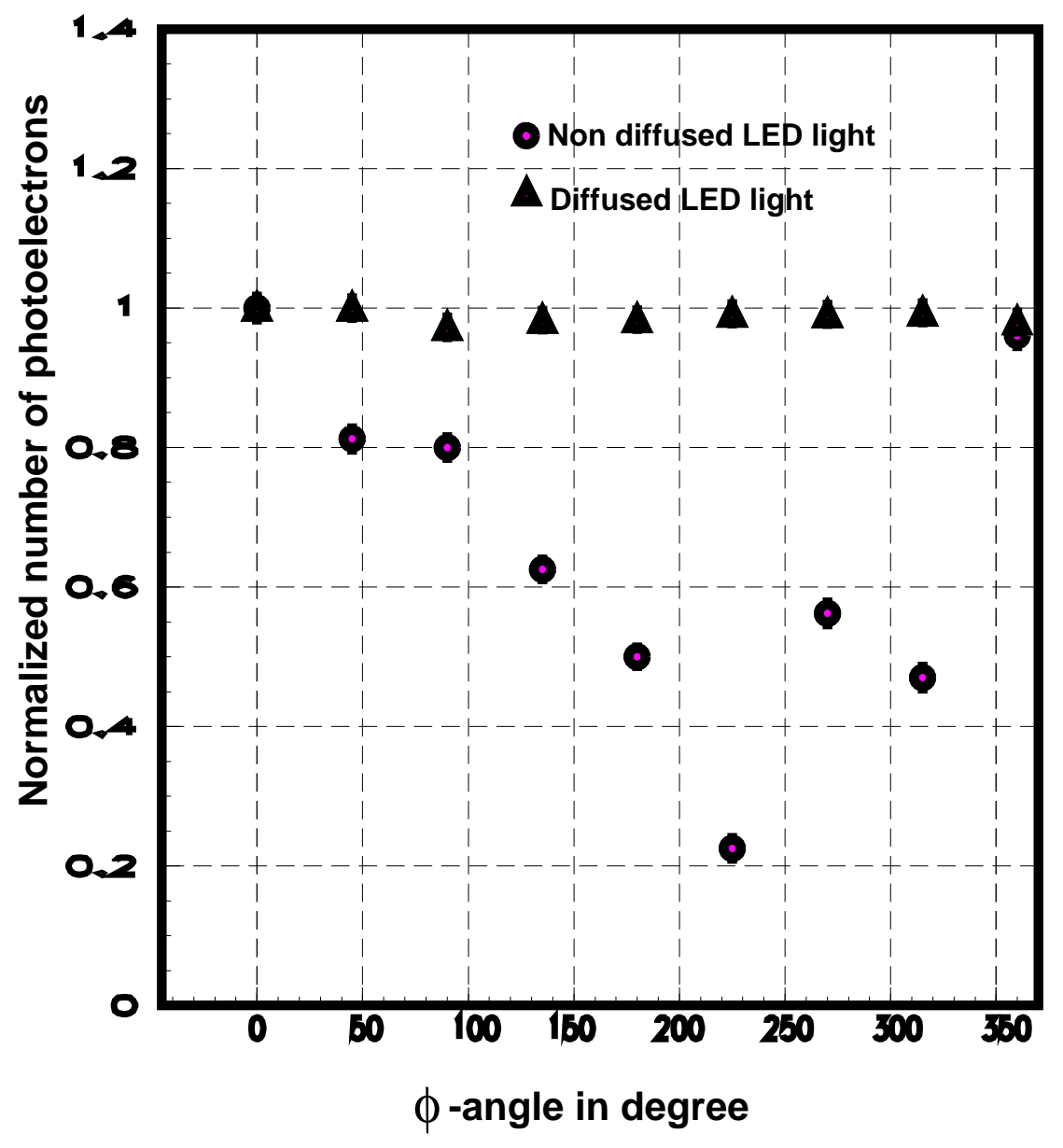

Figure 10: 


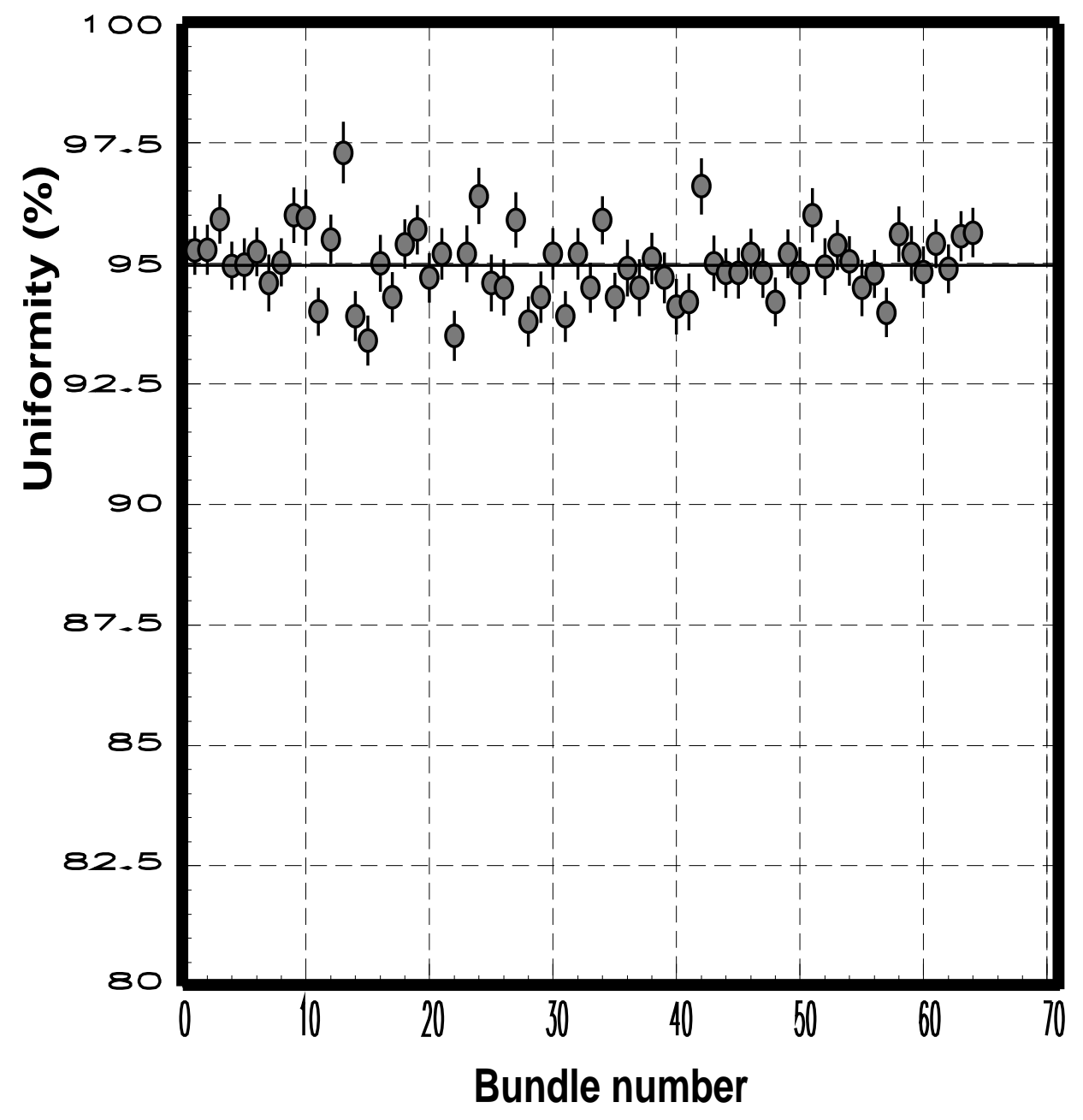

Figure 11: 


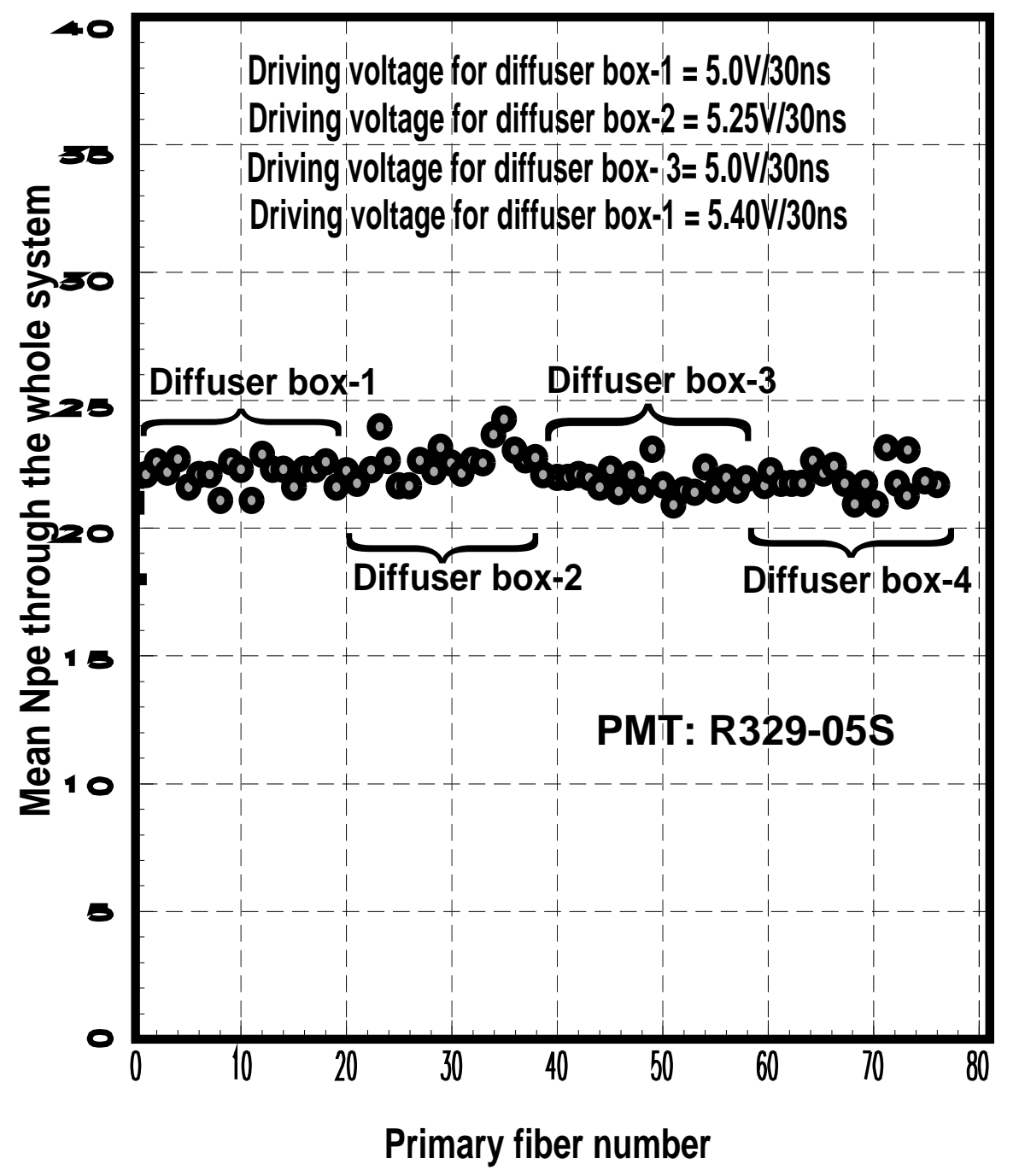

Figure 12: 


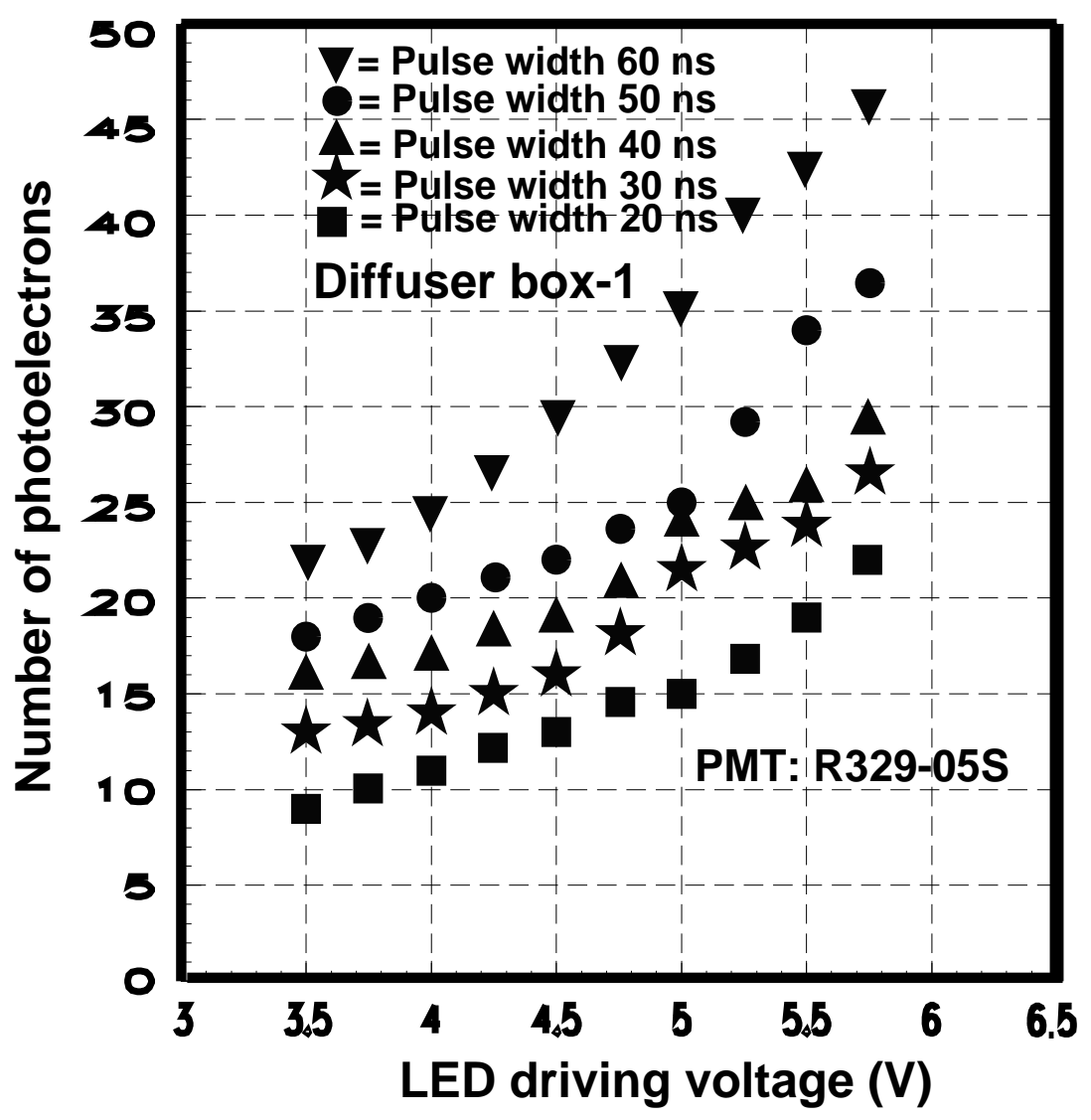

Figure 13: 